\title{
Arctigenin inhibits triple-negative breast cancers by targeting CIP2A to reactivate protein phosphatase $2 \mathrm{~A}$
}

\author{
QIUYUE HUANG ${ }^{1,3^{*}}$, SHANSHAN QIN ${ }^{1,2 *}$, XIAONING YUAN ${ }^{1,3}$, LIANG ZHANG $^{1,2}$, \\ JUANLI JI ${ }^{1,3}$, XUEWEN LIU ${ }^{1}$, WENJING MA ${ }^{1,3}$, YUNFEI ZHANG ${ }^{1,3}$, PENGFEI LIU ${ }^{1,3}$, \\ ZHITING SUN $^{1}$, JINGXUAN ZHANG ${ }^{1,2}$ and YING LIU ${ }^{1,2}$ \\ ${ }^{1}$ Laboratory of Molecular Target Therapy of Cancer, Institute of Basic Medical Sciences, \\ ${ }^{2}$ Department of Biochemistry, School of Basic Medical Sciences, and ${ }^{3}$ School of Biomedical Engineering, \\ Hubei University of Medicine, Shiyan, Hubei 442000, P.R. China
}

Received December 7, 2016; Accepted May 15, 2017

DOI: $10.3892 /$ or.2017.5667

\begin{abstract}
We have shown that a novel STAT3 inhibitor arctigenin (Atn) induces significant cytotoxicity in triple-negative breast cancer (TNBC) cells. This study further delineated molecular mechanisms where by Atn triggered cytotoxicity in TNBC cells. We found Atn can also inhibit metastasis in TNBC cells through cancerous inhibitor of protein phosphatase 2A (CIP2A) pathway. CIP2A is an endogenous inhibitor of protein phosphatase $2 \mathrm{~A}$ (PP2A), which can increase the migration and invasion of various cancer cells. PP2A is a tumor suppressor, which is functionally defective in various cancers. Atn-induced metastasis inhibition was associated with reactivation of $\mathrm{PP} 2 \mathrm{~A}$, downregulation of $\mathrm{CIP} 2 \mathrm{~A}$ and $\mathrm{Akt}$ phosphorylation. Silencing CIP2A enhanced Atn-induced metastasis inhibition and apoptosis in TNBCs. Furthermore, ectopic expression of CIP2A or inhibition of PP2A in TNBC cells abolished the effects of Atn. In conclusion, we found that enhancement of PP2A activity by inhibition of CIP2A, at least in part, promotes the anti-metastasis effect induced by Atn. Our findings disclose the novel therapeutic mechanism of this targeted agent, and suggest the therapeutic potential and feasibility of developing PP2A enhancers as a novel anticancer strategy.
\end{abstract}

\section{Introduction}

Breast cancer is the most frequently diagnosed cancer and the leading cause of cancer death among females worldwide, with

Correspondence to: Dr Ying Liu or Dr Jingxuan Zhang, Laboratory of Molecular Target Therapy of Cancer, Institute of Basic Medical Sciences, Hubei University of Medicine, Shiyan, Hubei 442000, P.R. China

E-mail: ying_liu1002@163.com

E-mail: zhangjx@hbmu.edu.cn

*Contributed equally

Key words: arctigenin, CIP2A, triple-negative breast cancers, PP2A, metastasis an estimated 1.7 million cases and 521,900 deaths in 2012 . Especially, less developed countries account for approximately one-half of all breast cancer cases and $62 \%$ of deaths (1). Breast cancer is also the leading cause of cancer death in women younger than 45 years in China (2); most of these fatalities are caused by metastatic disease. Approximately, 15-20\% of breast cancers are triple-negative breast cancer (TNBC) (3). TNBC is characterized by the absence of estrogen receptor, progesterone receptor, and HER-2 expression; this cancer results in high morbidity and mortality because of its rapid growth rate, metastatic potential, and frequently acquired treatment resistance (4). So far there is no FDA (Food and Drug Administration)-approved targeted therapy drug for TNBC patients. Considering that $<30 \%$ of women with metastatic TNBC survive 5 years (5), there is an urgent need to identify new therapy targets and illustrate the molecular events of TNBC.

Cancerous inhibitor of protein phosphatase 2A (CIP2A) is a cellular inhibitor of the important tumor-suppressor protein, protein phosphatase 2A (PP2A) (6). PP2A functions as a serine/threonine protein phosphatase that regulates several important oncogenic proteins such as Akt, ERK, c-Myc, and p70S6K $(7,8)$. When PP2A is inhibited by CIP2A, PP2Amediated dephosphorylation of these oncoproteins is blocked, thus promoting anchorage-independent cell growth and in vivo tumor formation. CIP2A functions as an oncoprotein that promotes proliferation and aggressiveness of several cancer types including head and neck squamous cell carcinoma, oral squamous cell carcinoma, esophageal squamous cell carcinoma, colon, gastric, breast, prostate, tongue, lung, and cervical cancer, as well as acute myeloid leukemia (6,9-12). In addition, aberrant expression, mutations, and somatic alterations of the PP2A scaffold and regulatory subunits are frequently found in human breast, lung, colon, and skin cancers (13). Therefore, reactivation of PP2A activity based on its tumor suppressor properties through inhibiting of CIP2A is considered to be an attractive therapeutic strategy for human cancer treatment $(14,15)$.

Arctigenin (Atn, Fig. 1) is a bioactive lignan isolated from the seeds of Arctium lappa L. Atn demonstrates antiinflammatory effects by inhibiting nuclear transcription 
factor- $\kappa \mathrm{B}(\mathrm{NF}-\kappa \mathrm{B})(16)$. Atn can also enhance the chemosensitivity of several cancer cells (HepG2, HeLa, and K562), to cisplatin by inhibiting the STAT3 signaling pathway at high doses (17). As we showed in our previous study (18), Atn inhibited cell growth and induced apoptosis in TNBCs by inhibiting STAT3. We used computational docking and molecular dynamics simulation showed that Atn had high-affinity interaction with the SH2 domain of STAT3 with residues (Arg688, Pro689, and Pro695). Atn inhibited STAT3 binding to genomic DNA by disrupting hydrogen bond binding between DNA and STAT3. However, whether Atn influences other pathways correlated to tumor aggressiveness and whether Atn functions as a multi-targeted drug requires further study.

In this study, we tested the effects of Atn on CIP2A expression in TNBC cells, and showed that Atn inhibits metastasis of TNBC cells with inhibition of CIP2A expression to reactivate PP2A activation. These data indicate that Atn is a potential multi-targeted drug for the treatment of TNBC.

\section{Materials and methods}

Reagents. Arctigenin (Atn) with a purity of $\leq 98 \%$ was purchased from Shanghai Yuanye Bio-Technology Co., Ltd. (Shanghai, China). Atn was dissolved in DMSO (Sigma-Aldrich, St. Louis, MO, USA) at a stock solution of $50 \mathrm{mM}$ and stored at $-20^{\circ} \mathrm{C}$. 3-(4,5)-dimethylthiazol(-z-y1)-3, 5-di-phenytetrazolium bromide (MTT) and okadaic acid (OA) were purchased from Sigma-Aldrich.

Cell culture. Human TNBC lines MDA-MB-231 and MDA-MB-468 were obtained from American Type Culture Collection (ATCC, Manassas, VA, USA), and maintained in Leibovitz's L-15 (Gibco; Thermo Fisher Scientific, Inc., Waltham, MA, USA) supplemented with 10\% FBS (Hyclone, GE Healthcare Life Sciences, Chalfont, UK) and antibiotics and incubated in a humidified atmosphere without $\mathrm{CO}_{2}$ at $37^{\circ} \mathrm{C}$.

Western blotting. Cell pellets were lysed in RIPA buffer containing $50 \mathrm{mM}$ Tris pH 8.0, $150 \mathrm{mM} \mathrm{NaCl}, 0.1 \%$ SDS, $0.5 \%$ deoxycholate, $1 \%$ NP-40, $1 \mathrm{mM}$ DTT, $1 \mathrm{mM} \mathrm{NaF}$, $1 \mathrm{mM}$ sodium vanadate, $1 \mathrm{mM}$ PMSF (Sigma-Aldrich, Merck Millipore, Darmstadt, Germany), and $1 \%$ protease inhibitors cocktail (Merck, Millipore). Lysates were normalized for total protein $(25 \mu \mathrm{g})$ and loaded on $8-12 \%$ sodium dodecyl sulfate polyacrylamide gel, electrophoresed, and transferred to a PVDF membrane (Millipore, Kenilworth, NJ, USA), followed by blocking with $5 \%$ skimmed milk at room temperature for $1 \mathrm{~h}$. The membrane was incubated with primary antibodies overnight at $4^{\circ} \mathrm{C}$ and rinsed with Tris-buffered saline with Tween-20. The primary antibodies used were anti-phosphoERK1/2 (Thr202/Tyr204) (1:1,000 dilation; cat. no. 9010), anti-ERK1/2 (1:1,000 dilation; cat. no. 9102), anti-PP2Ac (1:1,000 dilation; cat. no. 2038, anti-caspase-3 (1:1,000 dilation; cat. no. 9662), anti-caspase-9 (1:1,000 dilation; cat. no. 9508), anti-PARP (1:1,000 dilation; cat. no. 9542) (Cell Signaling Technology, Danvers, MA, USA), anti-CIP2A (1:500 dilation; cat. no. sc-80662), anti-phospho-Akt (Ser473) (1:500 dilation; cat. no. sc-7985), anti-Akt (1:500 dilation; cat. no. sc-8312), anti-CDK2 (1:500 dilation; cat. no. sc-6248) (Santa Cruz Biotechnology, Santa Cruz, CA, USA), and anti-GAPDH
(1:5,000 dilation; cat. no. M20006; Abmart, Shanghai, China) antibodies. The blots were then washed and incubated with horseradish peroxidase (HRP)-conjugated secondary antibody (1:10,000 dilation; cat. no. E030120-01 and E030110-01; EarthOx, LLC, San Francisco, CA, USA) at room temperature for $1.5 \mathrm{~h}$. Detection was performed by using a SuperSignal ${ }^{\circledR}$ West Pico Trial kit (cat. no. QA210131; Pierce Biotechnology, Inc., Rockford, IL, USA) (19). The defined sections of the film were scanned for image capture and quantification using Adobe Photoshop software (CS4, Adobe Systems Inc., USA) and ImageJ software (National Institutes of Health, Bethesda, MD, USA).

Reverse transcription-quantitative polymerase chain reaction (RT-qPCR). Expression of the CIP2A gene was examined by real-time polymerase chain reaction (RT-PCR) normalized to expression of GAPDH. Total RNA was extracted from cells using TRIzol reagent (Invitrogen; Thermo Fisher Scientific, Inc.) according to the manufacturer's protocol. RT-qPCR analysis of $C I P 2 A$ was performed with $2 \mu \mathrm{g}$ of total RNA and ReverTra Ace qPCR RT kit (Toyobo Co., Ltd. Life Science Department, Osaka Japan). Mixed $2 \mu \mathrm{g}$ RNA, $4 \mu \mathrm{l}$ 5X RT buffer, $1 \mu \mathrm{l}$ RT enzyme mix, $1 \mu \mathrm{l}$ primer mix, and nucleasefree water $\leq 20 \mu \mathrm{l}$ volume. The reverse transcription step was: $37^{\circ} \mathrm{C}$ for $15 \mathrm{~min} ; 98^{\circ} \mathrm{C}$ for $5 \mathrm{~min}$, then stored at $-20^{\circ} \mathrm{C}$. For RT-qPCR, we used CIP $2 A$ gene forward primer 5'-5'-TGCGGCACTTGGAGGTAATTTC-3', CIP2A gene reverse primer 5'-AGCTCTACAAGGCAACTCAAGC-3'; GAPDH forward primer 5'-TGTTGCCATCAATGACCC CTT-3', GAPDH reverse primer 5'-CTCCACGACGTACTCA GCG-3'. RT-qPCR was performed in an ABI StepOnePlus ${ }^{\mathrm{TM}}$ Real-Time PCR system (ABI; Thermo Fisher Scientifc, Inc.) using SYBR ${ }^{\circledR}$ Green Real-Time PCR Master Mix (Toyobo Co., Ltd. Life Science Department). Mixed SYBR Green PCR Master Mix $10 \mu \mathrm{l}$, forward and reverse primers $200 \mathrm{nM}$, cDNA template $100 \mathrm{ng}$, and $\mathrm{ddH}_{2} \mathrm{O} \leq 20 \mu \mathrm{l}$ volume. PCR conditions consisted of the following: $95^{\circ} \mathrm{C}$ for $3 \mathrm{~min}$ for denaturation; $95^{\circ} \mathrm{C}$ for $15 \mathrm{sec}$ for annealing; and $60^{\circ} \mathrm{C}$ for $1 \mathrm{~min}$ for extension, for 40 cycles. The threshold cycle for each sample was selected from the linear range and converted to a starting quantity by interpolation from a standard curve generated on the same plate for each set of primers. The CIP2A mRNA levels were normalized for each well to the GAPDH mRNA levels using the $2^{-\Delta \Delta C q}$ method (20). Each experiment was repeated three times.

PP2A activity assay. PP2A immunoprecipitation phosphatase assay kit (Upstate, Temecula, CA, USA) was used to measure phosphate release as an index of phosphatase activity according to the manufacturer's instructions. Briefly, $100 \mu \mathrm{g}$ protein isolated from cells was incubated with $4 \mu \mathrm{g}$ anti-PP $2 \mathrm{~A}$ monoclonal antibody overnight. Protein A agarose beads $(40 \mu \mathrm{l})$ were added and the mixture was incubated at $4^{\circ} \mathrm{C}$ for $2 \mathrm{~h}$. Subsequently, the beads were collected and washed three times with $700 \mu 1$ of ice-cold TBS and one time with $500 \mu 1$ Ser/Thr assay buffer. The beads were further incubated with $750 \mathrm{mM}$ phosphopeptide in assay buffer for $10 \mathrm{~min}$ at $30^{\circ} \mathrm{C}$ with constant agitation. Malachite Green Phosphate Detection solution $(100 \mu \mathrm{l})$ was added and the absorbance at $650 \mathrm{~nm}$ was measured on a microplate reader (21). 


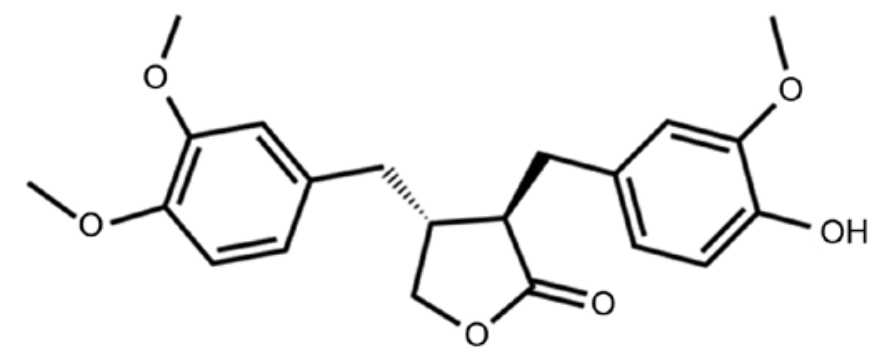

Figure 1. Chemical structure of arctigenin.

Cytotoxic assay and cell viability. Cells were seeded into 96-well plate and pre-cultured for $24 \mathrm{~h}$, then treated with Atn for $24 \mathrm{~h}$. Cell cytotoxicity was determined by MTT assay. The absorbance was measured at $570 \mathrm{~nm}$ by automated microplated reader (Bio-Tek, VT, USA), and the cell death rate was calculated as follows: Inhibition rate $(\%)=($ average $\mathrm{A}_{570}$ of the control group - average $\mathrm{A}_{570}$ of the experimentalgroup) / (average $A_{570}$ of the control group - average $A_{570}$ of the blank group) $\mathrm{x} 100 \%$. Cell viability was estimated by trypan blue dye exclusion (19).

Transfection of siRNA. Two siRNAs targeting CIP2A were designed and synthesized by Shanghai GenePharma Co., Ltd., (Shanghai, China) referred to as siRNA1 and siRNA2. The siRNA sequences were as follows: 5'-CUGUGGUUGUGU UUGCACUTT-3' (CIP2A siRNA1), 5'-ACCAUUGAUAUCC UUAGAATT-3' (CIP2A siRNA2), 5'-UUCUCCGAACGUG UCACGUTT-3' [negative control (NC) siRNA]. Using Lipofec tamine 2000 (Invitrogen; Thermo Fisher Scientific, Inc.) according to the manufacturer's protocol, TNBC cells were transfected with $100 \mathrm{nM}$ siRNA. Also, $48 \mathrm{~h}$ after transfection the cells were then harvested for western blotting, and cell viability.

Transfection of DNA. The pOTENT-1-CIP2A expression plasmid was purchased from Youbio Co. Ltd. (Changcha, China). Transfection of the the pOTENT-1-CIP2A plasmid into TNBC cells were carried out using Lipofectamine ${ }^{\circledR} 3000$ transfection reagent (Invitrogen; Thermo Fisher Scientific, Inc.) following the manufacturer's protocol.

Flow cytometric assays for Annexin $V(A V)$. Cell apoptosis was evaluated by AV detection using an AV-FITC kit (BD Biosciences, San Jose, CA, USA), according to the manufacturer's instructions (22).

Invasion assay. An invasion assay was carried out using 24-well plate (Corning). A polyvinyl-pyrrolidone-free polycarbonate filter (8- $\mu \mathrm{m}$ pore size) (Corning) was coated with Matrigel (BD). The lower chamber was filled with medium containing 20\% FBS as chemoattractant. The coated filter and upper chamber were laid over the lower chamber. Cells $\left(1 \times 10^{4}\right.$ cells/well) were preincubated with Atn for $30 \mathrm{~min}$ at room temperature, and then cell suspension containing Atn was seeded onto the upper chamber wells. After incubation for $20 \mathrm{~h}$ at $37^{\circ} \mathrm{C}$, the filter was fixed and stained with $2 \%$ ethanol containing $0.2 \%$ crystal violet $(15 \mathrm{~min})$. After being dried, the stained cells were enumerated under a light microscope at $x 10$ objective. For quantification, the invaded stained cells on the other side of the membrane were extracted with $33 \%$ acetic acid. The absorbance of the eluted stain was determined at $570 \mathrm{~nm}$.

Wound healing assay. Cells $\left(4 \times 10^{5}\right.$ cells $\left./ 2 \mathrm{ml}\right)$ were seeded in a 6-well plate and incubated at $37^{\circ} \mathrm{C}$ until $90-100 \%$ confluence. Then the confluent cells were scratched with a $200-\mu 1$ pipette tip, followed by washing with PBS, and then treated with Atn in a basic medium. After $24 \mathrm{~h}$ of incubation, the cells were fixed and stained with $2 \%$ ethanol containing $0.2 \%$ crystal violet powder (15 min), and randomly chosen fields were photographed under a light microscope at $4 \mathrm{X}$ objective. The number of cells migrated into the scratched area was calculated.

Statistical analysis. All experiments were repeated at least three times and the data are presented as the mean \pm SD unless noted otherwise. Differences between data groups were evaluated for significance using Student's t-test of unpaired data or one-way analysis of variance and Bonferroni post-test. P-values $<0.05$ indicate statistical significance.

\section{Results}

Atn downregulates CIP2A transcription and induces CIP2A proteolysis. In our previous study, we reported that CIP2A plays a critical role in the proliferation and aggressiveness of TNBC (23), and a natural compound oridonin is able to downregulate CIP2A in lung cancer cells (12). In this study, we found that, treatment with Atn at 0.4-1.2 $\mu \mathrm{M}$ for $24 \mathrm{~h}$ could downregulate CIP2A expression in TNBC cell lines MDA-MB-231 and MDA-MB-468 (Fig. 2A and B). As shown in Fig. 2A, the expression of CIP2A protein was reduced in MDA-MB-231 cells exposed to Atn at $0.4 \mu \mathrm{M}$ and became undetectable in cells treated with Atn at $1.2 \mu \mathrm{M}$ for $24 \mathrm{~h}$. Also, treatment of MDA-MB-468 cells with Atn at $0.8 \mu \mathrm{M}$ resulted in an apparent reduction of CIP2A (Fig. 2B). We further showed that Atn caused downregulation of CIP2A in a time-dependent manner (Fig. 2C and D). We next investigated whether Atn affected CIP2A gene transcription by RT-qPCR. We found that in response to Atn treatment for $24 \mathrm{~h}, \mathrm{CIP} 2 \mathrm{~A}$ was decreased drastically (Fig. 2E and F). These data indicate that Atn decreases CIP2A transcription. Since Atn caused a relatively rapid decrease of CIP2A, we hypothesized that Atn might also affect CIP2A stability. Thus, next we used a protein synthesis inhibitor cycloheximide (CHX) to block protein synthesis and then we found that CIP2A was stable under treatment with $\mathrm{CHX}$ for $>12 \mathrm{~h}$. However, it was downregulated in $6 \mathrm{~h}$ in cells coincubated with $\mathrm{CHX}$ and Atn (Fig. 2G and H). In conclusion, these results indicate that Atn downregulates $C I P 2 A$ transcription and induce CIP2A proteolysis.

Downregulation of CIP2A leads to increase of activated PP2A and decrease of phosphorylated Akt in TNSC cells. We next tested the expression of CIP2A downstream molecule Akt and found that Atn treatment downregulated Akt phosphorylation (pAkt) 
A

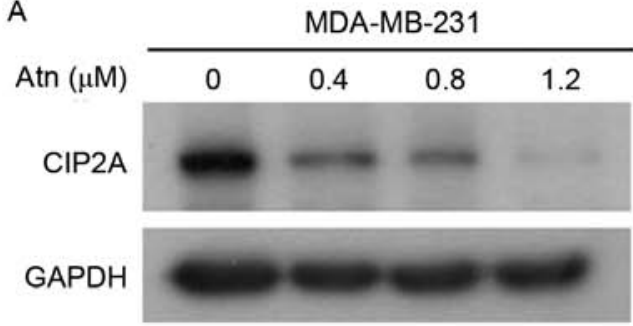

C

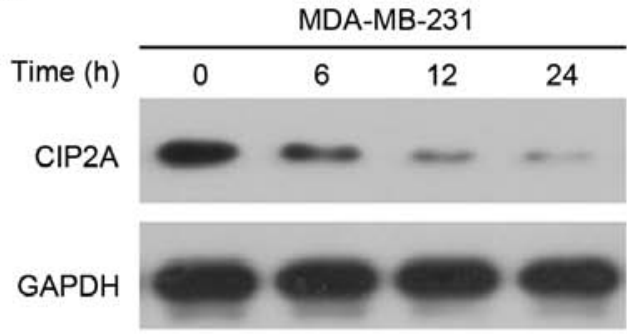

$E$

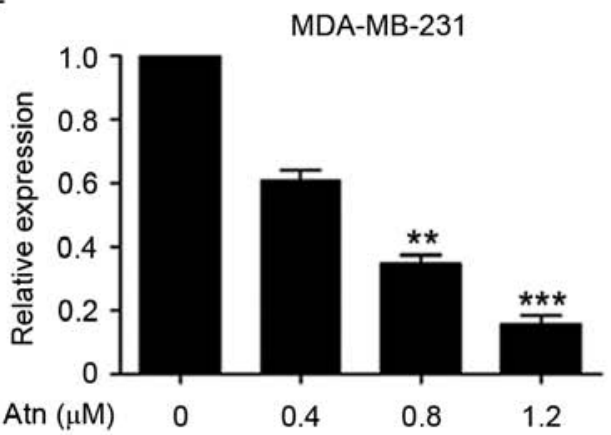

G

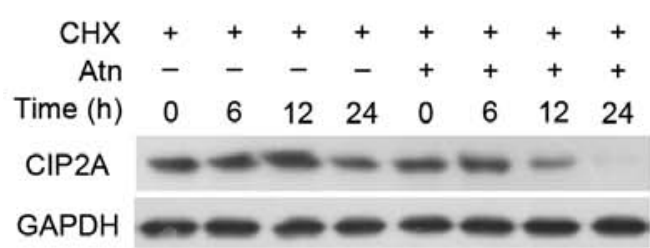

B

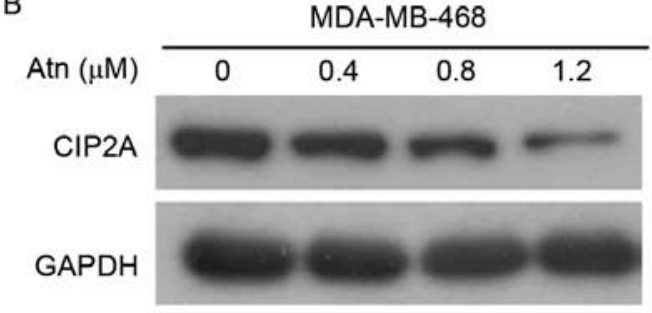

D

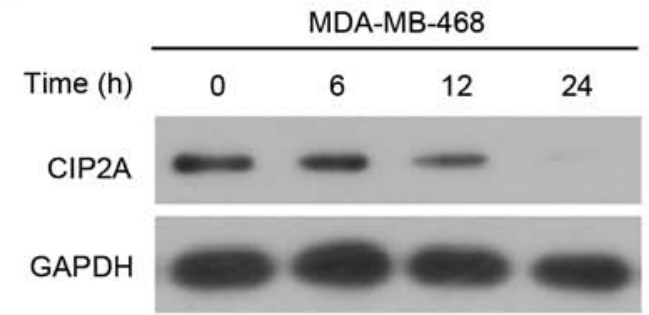

$\mathrm{F}$

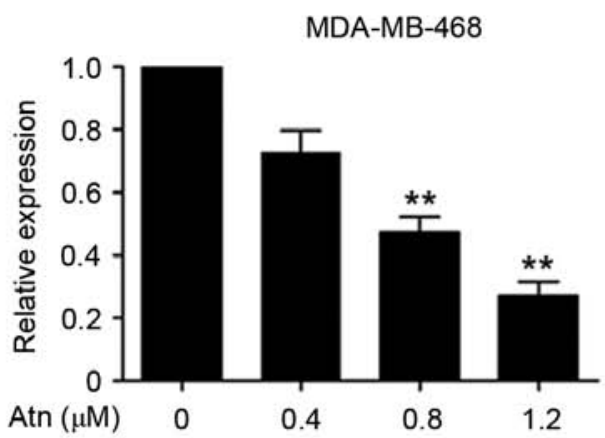

$\mathrm{H}$

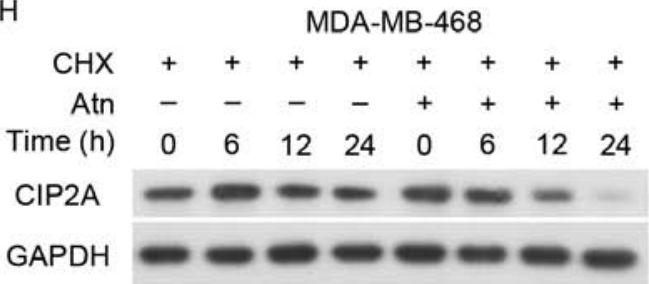

Figure 2. Atn downregulates CIP2A in TNBC cells. (A and B) MDA-MB-231 and MDA-MB-468 cells were treated with Atn at the indicated concentrations for $24 \mathrm{~h}$, followed by western blot analysis for indicated antibodies. GAPDH was used as a loading control. (C and D) MDA-MB-231 and MDA-MB-468 cells were treated with $0.4 \mu \mathrm{M}$ Atn for indicated time-points, and cell lysates were subjected to western blot assay. (E and F) The expression of CIP2A in cells upon Atn treatment for $24 \mathrm{~h}$ was analyzed by RT-qPCR. ${ }^{* * *} \mathrm{P}<0.01 \mathrm{vs} .0 \mu \mathrm{M}$. (G and $\left.\mathrm{H}\right)$ MDA-MB-231 and MDA-MB-468 cells were treated with $100 \mu \mathrm{g} / \mathrm{ml} \mathrm{CHX}$ in the absence or presence of $0.4 \mu \mathrm{M}$ Atn for indicated time-points, and cell lysates were subjected to western blot assay.

in both MDA-MB-231 (Fig. 3A) and MDA-MB-468 (Fig. 3B) cells, indicating Akt inactivation. CIP2A is an endogenous inhibitor of PP2A and the dephosphorylation of Akt is widely regulated by $\mathrm{PP} 2 \mathrm{~A}$, we therefore tested whether Atn could influence PP2A activity. As shown in Fig. 3C, Atn treatment has no effect on the expression of PP2Ac (catalytic subunit). We also tested another downstream molecule of PP2A, ERK, and found that Atn has no effect on ERK phosphorylation (pERK). Furthermore, we investigated PP2A activity and found that PP2A was upregulated in cells treated with Atn (Fig. 3D), suggesting that Atn may affect CIP2A/PP2A/pAkt signaling axis.
Targeting CIP2A/PP2A/pAkt axis promotes molecular mechanism of Atn-induced TNBC cell apoptosis. We subsequently examined whether CIP2A knockdown would alter cellular sensitivity to Atn. Two siRNAs targeting CIP2A were synthesized and applied to MDA-MB-231 and MDA-MB-468 cells with different Atn concentrations. Both siRNA1 and siRNA2 knockdown considerably decreased the expression of the CIP2A protein in each TNBC cell line (Fig. 4A and B). This finding validated the specificity and effectiveness of CIP2A siRNAs. To evaluate the role of CIP2A in Atn-induced proliferation inhibition and apoptosis, we transfected MDA-MB-231 and MDA-MB-468 cells with siRNA1 or siRNA2 targeting 
A

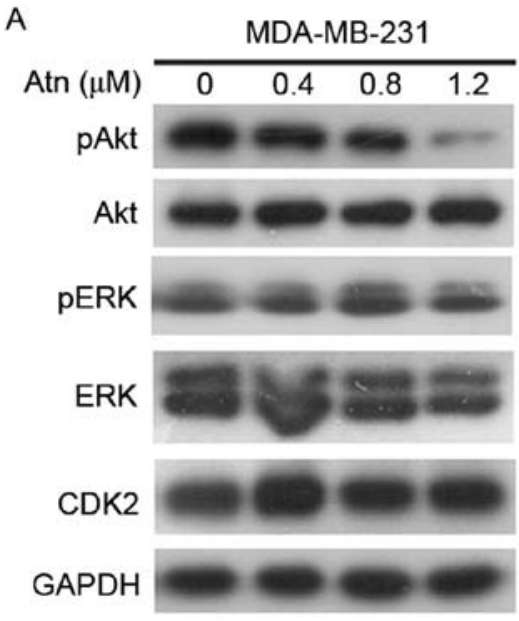

C

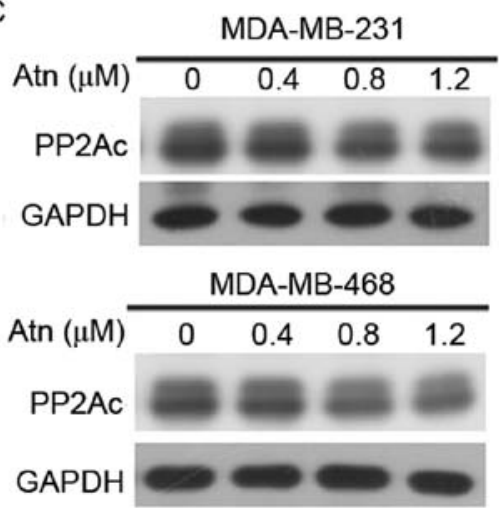

B

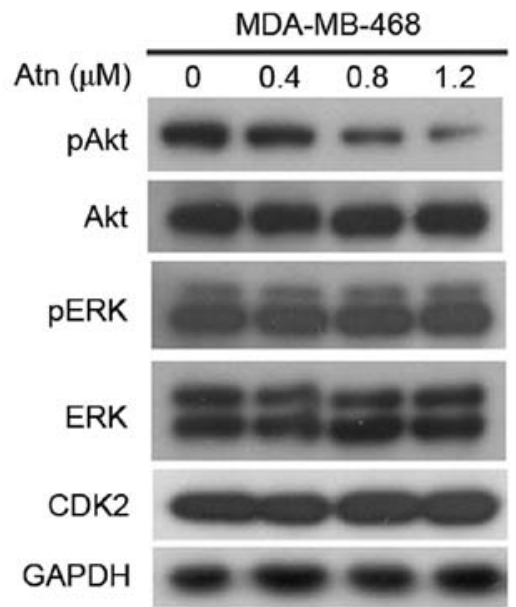

D

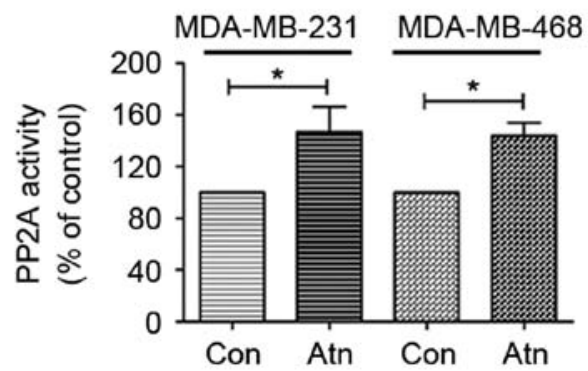

Figure 3. Effects of Atn on CIP2A downstream molecules. (A-C) MDA-MB-231 and MDA-MB-468 cells were treated with Atn at the indicated concentrations for $24 \mathrm{~h}$, followed by western blot analysis with the indicated antibodies. (D) MDA-MB-231 and MDA-MB-468 cells were treated with $1.2 \mu \mathrm{M}$ Atn for $24 \mathrm{~h}$ and lysed, and cell lysates were prepared for detecting PP2A activity as described in Materials and methods. ${ }^{*}<<0.05$.

CIP2A and then subjected to Atn treatment. Cell viability and western blot assays were used to detect variations in cell growth and protein expression. Notably, CIP2A silencing enhanced Atn-induced growth inhibition (Fig. 4C and D) and promoted Atn-induced apoptosis effects (Fig. 4E and F).

To further confirm the role of the CIP2A/PP2A/pAkt axis in mediating induction of apoptosis of TNBC cells by Atn, we generated MDA-MB-231 cells with ectopic overexpression of CIP2A by transient transfection (Fig. 4G). Compared with wild-type cells, the expression level of pAkt was upregulated in the CIP2A-overexpressing cells. Cell viability was used to detect variations in cell growth. Notably, CIP2A overexpression abolished Atn-induced growth inhibition (Fig. 4H) and inhibited Atn-induced apoptosis effects (Fig. 4I). These findings demonstrated that CIP2A, at least in part, plays a critical role in Atn-triggered TNBC proliferation inhibition. Atn also enhanced growth inhibition and apoptosis in CIP2A-silencing TNBC cells. Thus, these results confirmed that Atn induced cell proliferation inhibition and apoptosis, at least in part, by downregulating CIP2A.

Silencing CIP2A enhances Atn-inhibited invasive behavior of TNBC cells. Previous studies, including ours reported that CIP2A promoted the aggressiveness of breast cancer $(23,24)$. Next, we examined the effect of CIP2A depletion on Atn-inhibited invasive behavior. The invasion assay was performed in highly invasive MDA-MB-231 cells using Matrigel-coated 24-well microchemotaxis chambers. As shown in Fig. 5A, MDA-MB-231 cells were treated with CIP2A siRNA (100 nM) then Atn $(0.4 \mu \mathrm{M})$, and cell invasion was determined after 20 h. CIP2A depletion markedly suppressed the invasion and enhanced the effect of Atn. In addition, we explored the effect of CIP2A depletion on migration, MDA-MB-231 cells were treated with CIP2A siRNA $(100 \mathrm{nM})$ then Atn $(0.4 \mu \mathrm{M})$, and cell migration was determined after $48 \mathrm{~h}$. As shown in Fig. 5B, CIP2A depletion significantly decreased MDA-MB-231 cell migration and enhanced the effect of Atn.

Reactivation of PP2A is essential for Atn-inhibited proliferation and invasion. To evaluate whether Atn inhibited cell proliferation and invasion is due to reactivation of PP2A activity, we compared cell proliferation and invasion in Atn-treated cells in the presence and absence of PP2A inhibitor okadaic acid (OA). Our results showed that OA significantly reversed the cell proliferation and invasion inhibited by Atn (Fig. 6A and B). We next examined whether the inhibition of PP2A had any effect in the phosphorylation status of Akt, the target of PP2A. Consistent with our expectation, treatment of OA rescued Akt phosphorylation in Atn-treated cells. Similar results were also observed in MDA-MB-468 cells (Fig. 6C). Taken together, these 
A

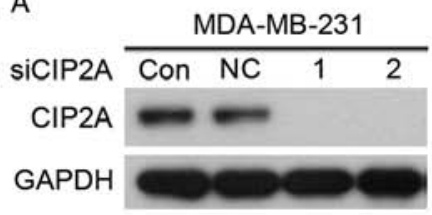

B

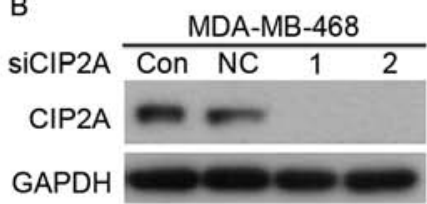

C

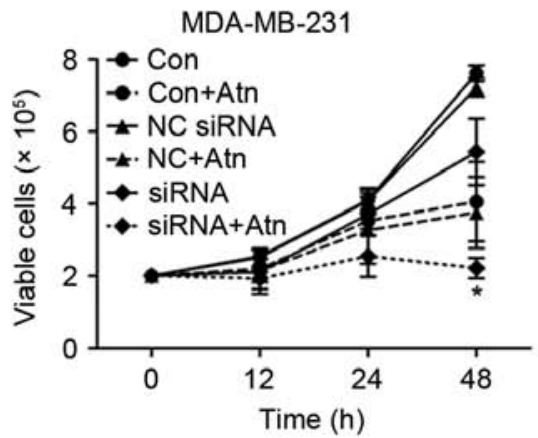

D

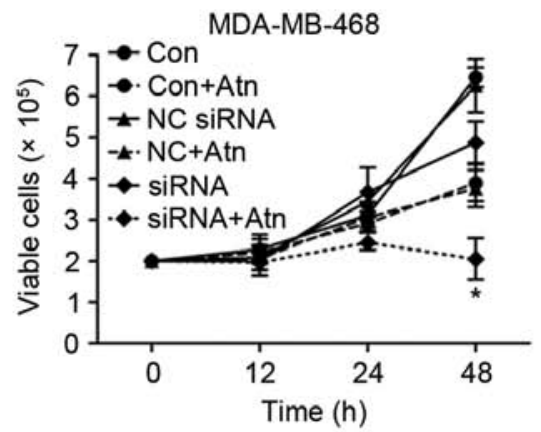

E

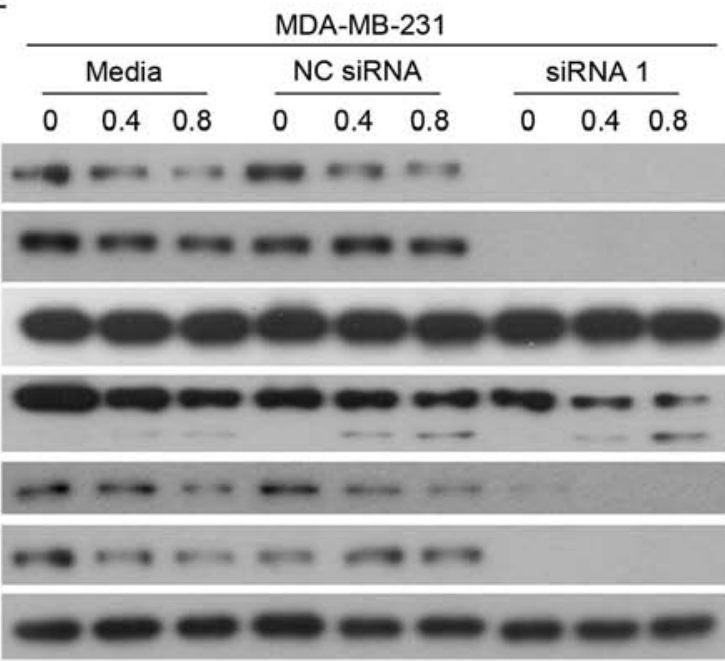

\begin{tabular}{|c|c|c|c|c|c|c|c|c|c|}
\hline \multirow[b]{3}{*}{ Atn $(\mu \mathrm{M})$} & \multicolumn{9}{|c|}{ MDA-MB-468 } \\
\hline & \multicolumn{3}{|c|}{ Media } & \multicolumn{3}{|c|}{ NC siRNA } & \multicolumn{3}{|c|}{ siRNA 1} \\
\hline & 0 & 0.4 & 0.8 & 0 & 0.4 & 0.8 & 0 & 0.4 & 0.8 \\
\hline CIP2A & - & $m$ & $\omega$ & 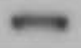 & $\rightarrow$ & $\Rightarrow$ & & & \\
\hline pAkt & $\cdots$ & mis & $a=$ & $m$ & & & & & \\
\hline Akt & & & & & & & & & \\
\hline PARP & & $=$ & 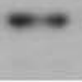 & $=$ & $m$ & - & $\cdots$ & $-\infty$ & $\cdots$ \\
\hline Pro-casp-3 & 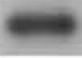 & $=$ & $=$ & $=$ & $\omega$ & $\omega$ & $=$ & $\Leftrightarrow$ & $m$ \\
\hline Pro-casp-9 & 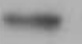 & - & $=$ & $=$ & & & & & \\
\hline GAPDH & & & & & & & & & \\
\hline
\end{tabular}

I
G

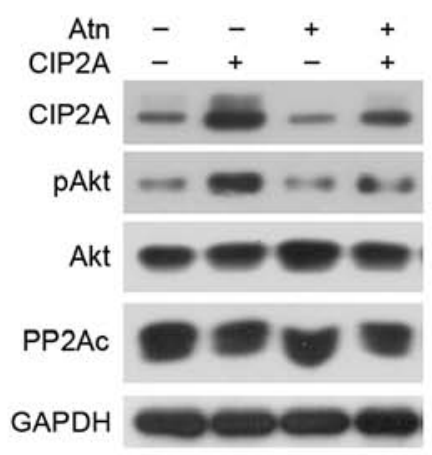

H

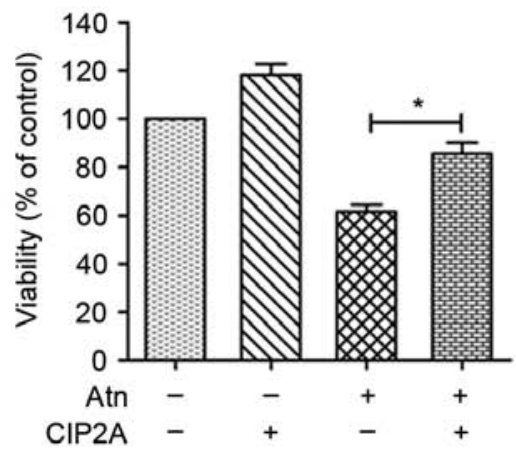

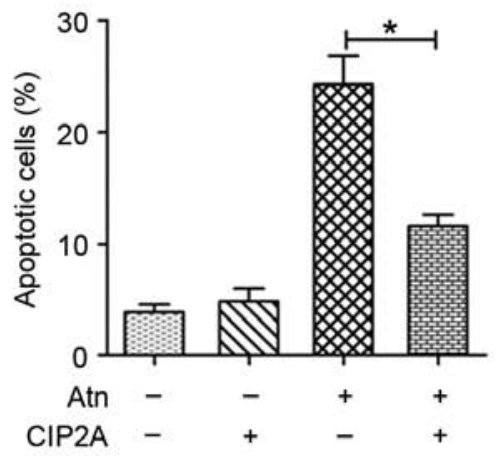

Figure 4. Targeting CIP2A/PP2A/pAkt pathway promotes molecular mechanism of Atn-induced TNBC cell apoptosis. (A and B) MDA-MB-231 and MDA-MB-468 cells were transfected with $100 \mathrm{nM} \mathrm{CIP2A-specific} \mathrm{siRNA} \mathrm{or} \mathrm{NC} \mathrm{siRNA} \mathrm{for} 48 \mathrm{~h}$. Cells were harvested for western blot analyses. (C and D) MDA-MB-231 and MDA-MB-468 cells were transfected with CIP2A-specific siRNA or NC siRNA, followed by treatment with Atn (0.4 $\mu$ M) for indicated times. (E and F) MDA-MB-231 and MDA-MB-468 cells were transfected with CIP2A-specific siRNA or NC siRNA, followed by treatment with Atn $(0,0.8$ and $1.2 \mu \mathrm{M})$ for $24 \mathrm{~h}$. Cells were harvested for western blot analyses. (G-I) MDA-MB-231 cells were transfected with a CIP2A expression plasmid, and then western blotting, MTT, and flow cytometry were used to detect proteins expression, proliferation, and apoptosis $48 \mathrm{~h}$ after transfection. ${ }^{*} \mathrm{P}<0.05$.

findings suggested that reactivation of PP2A is essential for Atn-inhibited proliferation and invasion via CIP2A inhibition.

\section{Discussion}

TNBC is a heterogeneous disease for which there is no targeted therapy as they do not express the respective drug targets (5). Moreover, TNBC is a highly aggressive form of breast cancer that is associated with early peak of recurrence, advanced stage at diagnosis and poorer outcome in comparison with non-TNBCs; in the advanced setting, responses to radiation therapy and chemotherapy lack durability. Finally, women who are diagnosed with TNBC tend to be younger than nonTNBC patients (25). Thus, there is an urgent need to develop a targeted therapy to inhibit the progression and metastasis of TNBC.

Our previous observations found that Atn inhibits proliferation and induces apoptosis in TNBCs cells by the 

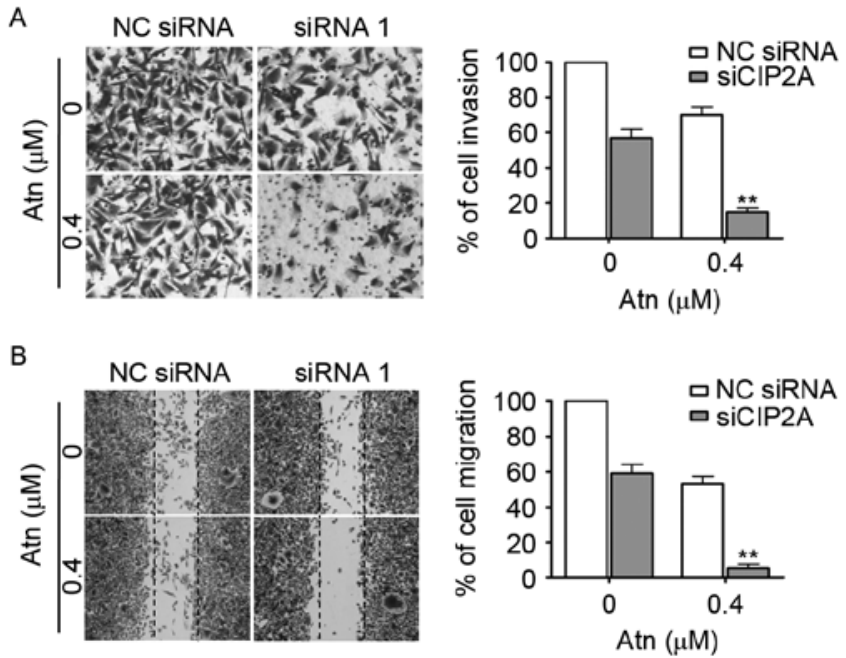

Figure 5. Silencing CIP2A enhances Atn-inhibited invasive behavior of TNBC cells. (A) MDA-MB-231 cells were transfected with $100 \mathrm{nM} \mathrm{CIP2A-specific}$ siRNA or NC siRNA, followed by treatment with Atn $(0.4 \mu \mathrm{M})$ for $24 \mathrm{~h}$. To evaluate cell invasion, the cells were analyzed for $20 \mathrm{~h}$ by invasion assay. (B) MDA-MB-231 cells were transfected with $100 \mathrm{nM}$ CIP2A-specific siRNA or NC siRNA, followed by treatment with Atn $(0.4 \mu \mathrm{M})$ for $24 \mathrm{~h}$. To evaluate cell migration, the cells were analyzed for $24 \mathrm{~h}$ by wound healing assay. ${ }^{* *} \mathrm{P}<0.01$ vs. NC siRNA. inhibition of STAT3 activation. Atn was identified to inhibit STAT3 binding to genomic DNA by disrupting hydrogen bond binding between DNA and STAT3. In addition, Atn augmented Taxotere ${ }^{\circledR}$-induced TNBC cell cytotoxicity (18). Here, we further showed that Atn was also able to cause a significant dose- and time-dependent decrease of CIP2A at both mRNA and protein levels in TNBCs (Fig. 2).

We tested the expression of CIP2A downstream molecule Akt and found that Atn treatment downregulated pAkt (but not total Akt) in both MDA-MB-231 (Fig. 3A) and MDA-MB-468 (Fig. 3B) cells. CIP2A is an endogenous inhibitor of PP2A and the dephosphorylation of Akt is widely regulated by PP2A. Next, we detected PP2A phosphatase activity and found that Atn significantly upregulated PP2A activity in both MDA-MB-231 and MDA-MB-468 cells (Fig. 3D). PP2A is a serine/threonine phosphatase that has a critical role in regulating various cellular processes, including signaling transduction, protein synthesis, cell cycle determination, metabolism, apoptosis and stress response (26). Because loss of PP2A function has been identified in various malignant diseases such as cancer of the lung, liver, colon, and breast, it has been reported that enhancing PP2A activity could be an effective approach for cancer treatment (27). Thus,
A

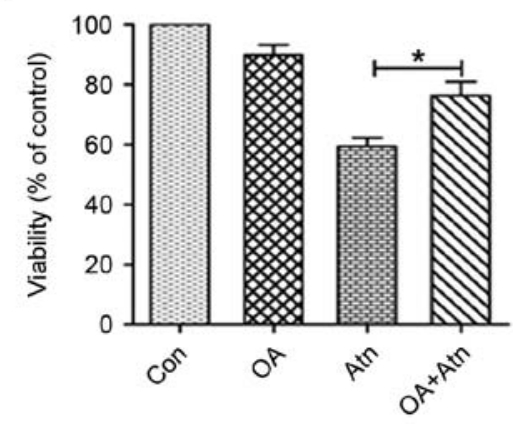

B

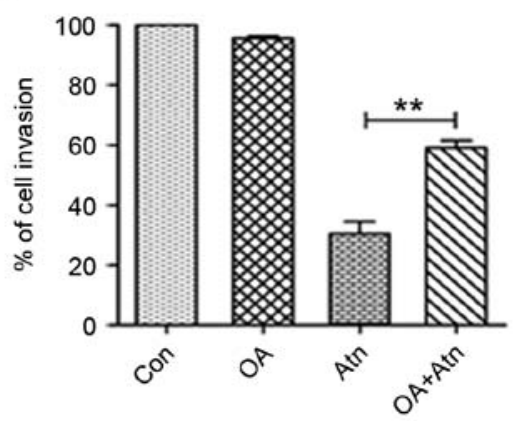

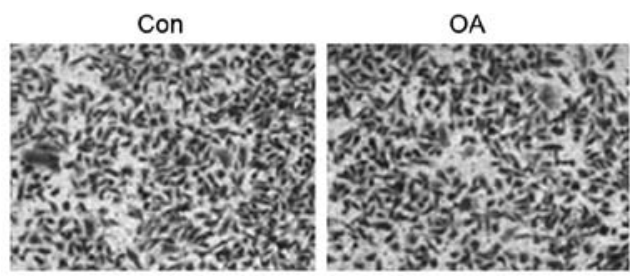

Atn

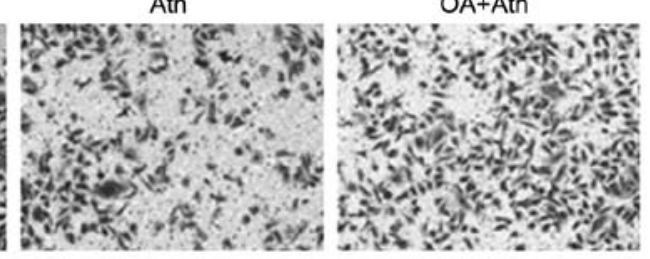

C
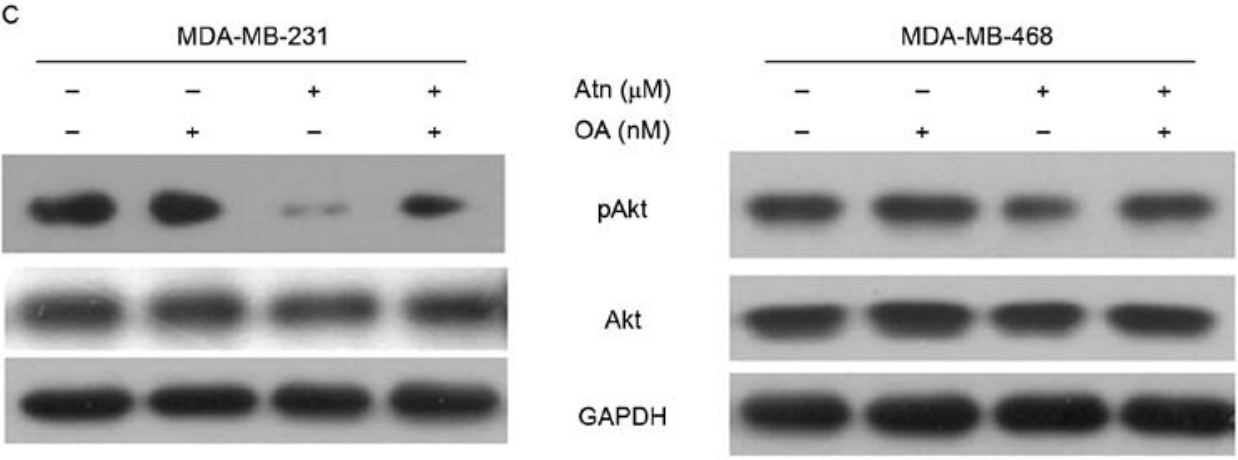

Figure 6. Reactivation of PP2A is essential for Atn-inhibited proliferation and invasion. (A) MDA-MB-231 cells were treated with Atn (1.2 $\mu \mathrm{M})$ and/or OA $(50 \mathrm{nM})$ for $24 \mathrm{~h}$ and analyzed by MTT assay. (B) MDA-MB-231 cells were treated with Atn $(0.4 \mu \mathrm{M})$ and/or OA (50 nM). To evaluate cell invasion, the cells were analyzed for $20 \mathrm{~h}$ by invasion assay. (C) MDA-MB-231 and MDA-MB-468 cells were treated with Atn $(0.4 \mu \mathrm{M})$ and/or OA (50 $\mathrm{nM})$ for $24 \mathrm{~h}$ and analyzed by western blotting. ${ }^{*} \mathrm{P}<0.05,{ }^{* * *} \mathrm{P}<0.01$. 
we showed that by Atn-induced inhibition of CIP2A, activity of PP2A was significantly enhanced and expression of pAkt was downregulated in TNBCs cells.

Moreover, we demonstrated that knockdown of CIP2A enhanced the proliferation inhibition and apoptosis effect of Atn in TNBCs and activated PP2A (Fig. 4C-F). Furthermore, ectopic expression of CIP2A upregulated the expression of pAkt (Fig. 4G), indicating that PP2A was inactivated and then Akt was activated. Then, we examined whether ectopic expressed CIP2A affected the treatment of Atn. Atn can downregulate CIP2A expression in MDA-MB-231 cell overexpressed CIP2A (Fig. 4G). We also detected proliferation and apoptosis of MDA-MB-231 cell overexpressed CIP2A (Fig. 4H and I), and found that CIP2A overexpression overcame the proliferation inhibition and apoptosis effect of Atn. These data validated the mechanism by which Atn-induced cancer cell apoptosis in TNBC cells, that is, induction of cancer cell apoptosis, at least in part, by inhibiting CIP2A and enhancing pAkt downregulation.

Migration and invasion are two important prerequisites of TNBC cancer progression and metastasis. Our previous research showed that $\mathrm{CIP} 2 \mathrm{~A}$ depletion markedly inhibited the invasive and migratory abilities of MDA-MB-231 cells. We investigated whether CIP2A depletion could enhance the effects of Atn in MDA-MB-231 cell line and found that silencing CIP2A significantly enhanced Atn-induced migration and invasion inhibition in MDA-MB-231 cells (Fig. 5). To further investigate the effect of PP2A activation in Atn induced TNBC proliferation and invasion inhibition, we co-incubated OA, the inhibitor of PP2A, with Atn in TNBC cells. The results showed that loss of $\mathrm{PP} 2 \mathrm{~A}$ function can antagonize the inhibition effect of Atn in TNBC cell proliferation and invasion (Fig. 6A and B) through Akt pathway (Fig. 6C). These results confirmed that $\mathrm{PP} 2 \mathrm{~A}$ reactivation plays a critical role in Atn induced CIP2A inhibition of downregulation mediated TNBC proliferation and invasion.

In conclusion, we demonstrated the Atn novel therapeutic mechanism in TNBC, that is, enhancement of PP2A-dependent pAkt downregulation by inhibition of CIP2A. Our results showed that designing agents to reactivate PP2A may be a feasible strategy for the development of novel TNBC therapies. Further studies on the detailed molecular modification of the CIP2A/PP2A/Akt signaling axis by Atn and exploring its possible application in other cancers are warranted.

\section{Acknowledgements}

This study was supported by grants from the National Natural Sciences Foundation of China (grant no. 81400157); the Natural Science Foundation of Hubei Provincial Department of Education (grant nos. Q20152106 and Q20162114); the Natural Science Foundation of Hubei Province of China (grant nos. 2016CFB528 and 2015CFB212); the Foundation of Health and Family planning Commission of Hubei Province (grant nos. WJ2015MB291 and WJ2017F065, 067); the Faculty Development Grants from Hubei University of Medicine (grant nos. 2014QDJZR06, 2014QDJZR08 and 2015QDJZR16); the Foundation of Hubei University of Medicine (grant nos. FDFR201605 and FDFR201609); and the Key Discipline Project of Hubei University of Medicine.

\section{References}

1. Torre LA, Bray F, Siegel RL, Ferlay J, Lortet-Tieulent J and Jemal A: Global cancer statistics, 2012. CA Cancer J Clin 65: 87-108, 2015

2. Chen W, Zheng R, Baade PD, Zhang S, Zeng H, Bray F, Jemal A, $\mathrm{Yu}$ XQ and He J: Cancer statistics in China, 2015. CA Cancer J Clin 66: 115-132, 2016.

3. Wang ZT, Chen ZJ, Jiang GM, Wu YM, Liu T, Yi YM, Zeng J, Du $\mathbf{J}$ and Wang HS: Histone deacetylase inhibitors suppress mutant p53 transcription via HDAC $8 / Y$ Y1 signals in triple negative breast cancer cells. Cell Signal 28: 506-515, 2016.

4. Deng XS, Wang S, Deng A, Liu B, Edgerton SM, Lind SE, Wahdan-Alaswad R and Thor AD: Metformin targets Stat 3 to inhibit cell growth and induce apoptosis in triple-negative breast cancers. Cell Cycle 11: 367-376, 2012.

5. Shu S, Lin CY, He HH, Witwicki RM, Tabassum DP, Roberts JM, Janiszewska M, Huh SJ, Liang Y, Ryan J, et al: Response and resistance to BET bromodomain inhibitors in triple-negative breast cancer. Nature 529: 413-417, 2016.

6. Junttila MR, Puustinen P, Niemelä M, Ahola R, Arnold H, Böttzauw T, Ala-aho R, Nielsen C, Ivaska J, Taya Y, et al: CIP2A inhibits PP2A in human malignancies. Cell 130: 51-62, 2007.

7. Rincón R, Cristóbal I, Zazo S, Arpí O, Menéndez S, Manso R, Lluch A, Eroles P, Rovira A, Albanell J, et al: PP2A inhibition determines poor outcome and doxorubicin resistance in early breast cancer and its activation shows promising therapeutic effects. Oncotarget 6: 4299-4314, 2015.

8. Tsukamoto S, Huang Y, Umeda D, Yamada S, Yamashita S, Kumazoe M, Kim Y, Murata M, Yamada K and Tachibana H: $67-\mathrm{kDa}$ laminin receptor-dependent protein phosphatase $2 \mathrm{~A}$ (PP2A) activation elicits melanoma-specific antitumor activity overcoming drug resistance. J Biol Chem 289: 32671-32681, 2014.

9. Li W, Ge Z, Liu C, Liu Z, Björkholm M, Jia J and Xu D: CIP2A is overexpressed in gastric cancer and its depletion leads to impaired clonogenicity, senescence, or differentiation of tumor cells. Clin Cancer Res 14: 3722-3728, 2008.

10. Ren J, Li W, Yan L, Jiao W, Tian S, Li D, Tang Y, Gu G, Liu H and $\mathrm{Xu} \mathrm{Z}$ : Expression of CIP2A in renal cell carcinomas correlates with tumour invasion, metastasis and patients' survival. Br J Cancer 105: 1905-1911, 2011.

11. Liu CY, Shiau CW, Kuo HY, Huang HP, Chen MH, Tzeng CH and Chen KF: Cancerous inhibitor of protein phosphatase 2A determines bortezomib-induced apoptosis in leukemia cells. Haematologica 98: 729-738, 2013.

12. Xiao X, He Z, Cao W, Cai F, Zhang L, Huang Q, Fan C, Duan C, Wang X, Wang J, et al: Oridonin inhibits gefitinib-resistant lung cancer cells by suppressing EGFR/ERK/MMP-12 and CIP2A/ Akt signaling pathways. Int J Oncol 48: 2608-2618, 2016.

13. Seshacharyulu P, Pandey P, Datta K and Batra SK: Phosphatase: PP2A structural importance, regulation and its aberrant expression in cancer. Cancer Lett 335: 9-18, 2013.

14. Schönthal AH: Role of serine/threonine protein phosphatase $2 \mathrm{~A}$ in cancer. Cancer Lett 170: 1-13, 2001.

15. Lv P, Wang Y, Ma J, Wang Z, Li JL, Hong CS, Zhuang Z and Zeng YX: Inhibition of protein phosphatase $2 \mathrm{~A}$ with a small molecule LB100 radiosensitizes nasopharyngeal carcinoma xenografts by inducing mitotic catastrophe and blocking DNA damage repair. Oncotarget 5: 7512-7524, 2014.

16. Cho MK, Park JW, Jang YP, Kim YC and Kim SG: Potent inhibition of lipopolysaccharide-inducible nitric oxide synthase expression by dibenzylbutyrolactone lignans through inhibition of I-kappaBalpha phosphorylation and of p65 nuclear translocation in macrophages. Int Immunopharmacol 2: 105-116, 2002.

17. Yao X, Zhu F, Zhao Z, Liu C, Luo L and Yin Z: Arctigenin enhances chemosensitivity of cancer cells to cisplatin through inhibition of the STAT3 signaling pathway. J Cell Biochem 112: 2837-2849, 2011.

18. Feng T, Cao W, Shen W, Zhang L, Gu X, Guo Y, Tsai HI, Liu X, Li J, Zhang J, et al: Arctigenin inhibits STAT3 and exhibits anticancer potential in human triple-negative breast cancer therapy. Oncotarget 8: 329-344, 2017.

19. Cao W, Liu Y, Zhang R, Zhang B, Wang T, Zhu X, Mei L, Chen H, Zhang $\mathrm{H}$, Ming P, et al: Homoharringtonine induces apoptosis and inhibits STAT3 via IL-6/JAK1/STAT3 signal pathway in Gefitinib-resistant lung cancer cells. Sci Rep 5: 8477, 2015.

20. Livak KJ and Schmittgen TD: Analysis of relative gene expression data using real-time quantitative PCR and the 2(-Delta Delta C(T)) method. Methods 25: 402-408, 2001. 
21. Liu H, Gu Y, Wang H, Yin J, Zheng G, Zhang Z, Lu M, Wang C and He Z: Overexpression of PP2A inhibitor SET oncoprotein is associated with tumor progression and poor prognosis in human non-small cell lung cancer. Oncotarget 6: 14913-14925, 2015.

22. Liu Y, Dong Y, Zhang B and Cheng YX: Small compound 6-O-angeloylplenolin induces caspase-dependent apoptosis in human multiple myeloma cells. Oncol Lett 6: 556-558, 2013.

23. Li S, Feng TT, Guo Y, Yu X, Huang Q, Zhang L, Tang W and Liu Y: Expression of cancerous inhibitor of protein phosphatase $2 \mathrm{~A}$ in human triple negative breast cancer correlates with tumor survival, invasion and autophagy. Oncol Lett 12: 5370-5376, 2016.

24. Come C, Laine A, Chanrion M, Edgren H, Mattila E, Liu X, Jonkers J, Ivaska J, Isola J, Darbon JM, et al: CIP2A is associated with human breast cancer aggressivity. Clin Cancer Res 15 5092-5100, 2009.
25. Wright HJ, Arulmoli J, Motazedi M, Nelson LJ, Heinemann FS, Flanagan LA and Razorenova OV: CDCP1 cleavage is necessary for homodimerization-induced migration of triple-negative breast cancer. Oncogene 35: 4762-4772, 2016.

26. Cristobal I, Rincon R, Manso R, Caramés C1, Zazo S2, MadozGúrpide J2, Rojo F3 and García-Foncillas J: Deregulation of the PP2A inhibitor SET shows promising therapeutic implications and determines poor clinical outcome in patients with metastatic colorectal cancer. Clin Cancer Res 21: 347-356, 2015.

27. Yu HC, Hung MH, Chen YL, Chu PY, Wang CY, Chao TT, Liu CY, Shiau CW and Chen KF: Erlotinib derivative inhibits hepatocellular carcinoma by targeting CIP2A to reactivate protein phosphatase 2A. Cell Death Dis 5: e1359, 2014. 